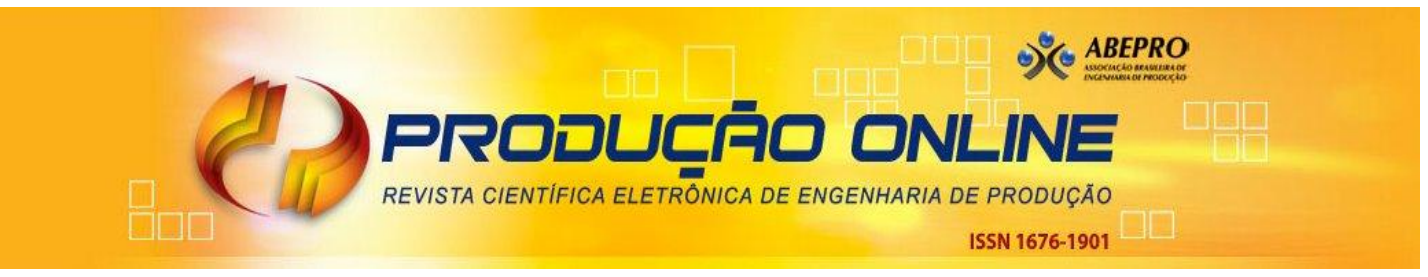

\title{
PRODUÇÃO SUCROALCOOLEIRA: ESTRATÉGIAS FINANCEIRAS E OPERACIONAIS
}

\section{PRODUCTION OF SUGAR AND ALCOHOL: FINANCIAL AND OPERATIONAL STRATEGIES}

\author{
Celma de Oliveira Ribeiro*E-mail:celma@usp.br \\ Sydnei Marssal de Oliveira** E-mail: sydnei.oliveira@fgv.br \\ Thiago de Oliveira Mendes*** E-mail: thiago.mendes@baml.com \\ *Escola Politécnica da Universidade de São Paulo (EPUSP), São Paulo, SP \\ ${ }^{* *}$ Fundação Getúlio Vargas (EAESP), São Paulo, SP \\ ${ }^{* * *}$ Banc of America Securities - Merrill Lynch, São Paulo, SP
}

\begin{abstract}
Resumo: Este artigo propõe a construção de um modelo de otimização para a definição do portfólio de produtos de uma usina sucroalcooleira, levando em conta aspectos operacionais e financeiros. Considera-se que a receita obtida por um produtor provém da venda de açúcar e álcool no mercado físico e de resultados obtidos através de operações de hedge no mercado de derivativos de açúcar. Empregando como medida de risco o CVaR (Conditional Value-at-Risk), o modelo possibilita a construção de uma fronteira eficiente e, de acordo com a tolerância ao risco do produtor, define a estratégia ideal de produção (mix de produção) e de atuação no mercado de derivativos (razão de hedging). Através do modelo o artigo busca também analisar a vantagem do uso do mercado de opções na construção de estratégias financeiras de hedging em mercados de commodities agrícolas.
\end{abstract}

Palavras-chave: Setor sucroalcooleiro. Mix de Produção. Razão de hedge. Otimização. Gestão de Portfólio.

Abstract: This article proposes the construction of an optimization model to define the product portfolio of a sugarcane mill, taking into account operational and financial aspects. It is considered that the revenue earned by a producer comes from the sale of sugar and alcohol in the physical market and the results obtained through hedging in the derivatives market of sugar. Employing CVaR (Conditional Value-at-Risk), as the risk measure, the model allows the construction of an efficient frontier and, according to the producer's risk tolerance, defines the optimal strategy of production (production mix) and activity in the derivatives market (hedge ratio). Through the model the article also seeks to analyze the advantage of using the options market in the construction of financial hedging strategies in agricultural commodities markets.

Keywords: Sugar Alcohol. Production Mix. Hedge Ratio. Optimization. Portfolio Selection.

\section{INTRODUÇÃO}

O mercado de commodities agrícolas conta com inúmeros instrumentos que possibilitam reduzir o risco financeiro a que os produtores agrícolas estão sujeitos. Em especial o mercado de derivativos possibilita a construção de estratégias financeiras com maior ou menor complexidade, sendo bastante conhecidas as que

Revista Produção Online, Florianópolis, SC, v.14, n. 4, p. 1270-1291, out./dez. 2014. 
utilizam o mercado futuro e de opções. Entretanto as estratégias financeiras e de produção não costumam ser tomadas de maneira agregada, de tal forma que a partir da definição ad hoc do mix de produção, compra-se (ou vende-se) ativos no mercado futuro ou de opções visando assegurar preços. Uma pergunta importante refere-se, portanto à possibilidade de determinar o mix de produção em conjunto com as estratégias no mercado de derivativos. Este problema é particularmente relevante em situações nas quais há flexibilidade de produção, como ocorre na produção sucroalcooleira, em que o usineiro pode - a partir da mesma matéria prima - produzir açúcar e álcool combustível.

Desta forma o objetivo deste artigo é apresentar um modelo de decisão para construção de um portfólio de produtos, de modo a considerar a flexibilidade gerada pela opção entre diferentes produtos agrícolas oriundos da mesma matéria prima. $\mathrm{O}$ modelo considera as operações no mercado físico e as operações financeiras realizadas no mercado futuro e de opções com a finalidade de proteção ou hedge. Para estudar este problema optou-se por considerar o mercado sucroalcooleiro, tanto pela sua importância em âmbito nacional e internacional, quanto pela disponibilidade de um mercado de derivativos consolidado.

Com base no modelo proposto pretende-se também avaliar se a proposição de LAPAN et al. (1991) é aplicável ao mercado sucroalcooleiro brasileiro. Os autores afirmam que quando o preço futuro não está viesado, as opções são instrumentos de hedging redundantes.

BASTIAN-PINTO et al. (2009) calculam o valor da opção gerada pela flexibilidade de se produzir açúcar ou etanol, levando em consideração preços do mercado brasileiro, através de um modelo de preços por reversão à média. Contudo esse trabalho não apresenta um modelo de decisão que considere uma medida de risco coerente, como o Valor em Risco Condicional (CVaR), nem mecanismos de controle do risco através de hedge no mercado futuro ou de opções como é feito no modelo aqui apresentado.

A literatura recente tem buscado tratar da questão de hedging em mercados de commodities. AITSAHLIA et al. (2009) investigam o impacto de previsões climáticas no planejamento do plantio e nas estratégias de hedge com contratos futuros. Nesse trabalho é utilizado o CVaR como medida de risco e uma cópula gaussiana para modelar cenários com uma distribuição conjunta de retornos e 
preços. O modelo é aplicado a produção de algodão no sudeste dos Estados Unidos, porém os autores não consideram a possibilidade de diversificação da produção.

Modelos que consideram decisões de produção, investimento, comercialização e proteção financeira de commodities, mesmo as não agrícolas e não diversificáveis são raros na literatura, sendo o trabalho mais notável o de Hélyette Geman (GEMAN \& OHANA, 2008). O modelo proposto pela autora permite gerar uma estratégia ótima de investimento, multiperíodo, em um portfólio de commodities estocáveis, sendo consistente no tempo, levando em consideração a posse da mercadoria física, e posições em contratos à vista e futuros, além da consideração da função utilidade para modelar a aversão ao risco e elasticidade temporal de substituição. No entanto, o modelo proposto não considera a possibilidade de diversificação da produção.

Os modelos para decisões de produção de commodities agrícolas diversificáveis, em geral, tratam da cadeia de suprimentos e consideram a demanda como informação de entrada, ou seja, não considera o andamento dos preços nas decisões de produção, esse é o caso de EBADIAN et al. (2013), VAN DYKEN et al. (2010) e Zhang et al. (2013)

O modelo aqui proposto baseia-se no trabalho de ALEXANDER et al. (2006) para quantificar o retorno das operações de hedging no mercado de derivativos de açúcar e, com isso, tornar possível a definição da razão de hedge. A função objetivo utilizada busca maximizar a utilidade, seguindo conceitos apresentados por CHEN et al. (2008).

As principais contribuições do artigo são:

1. A criação de um modelo capaz de construir a fronteira eficiente para um produtor de açúcar e álcool, ou seja, um modelo capaz de definir o mix de produção e a razão de hedge ideais, de acordo com a tolerância ao risco do produtor.

2. A análise sobre o impacto causado pela utilização de opções como instrumentos de hedge na fronteira eficiente de um produtor, quando comparado com o uso exclusivo do mercado futuro. Ou seja, busca-se analisar em que condições o portfólio ótimo deve incluir opções. 
O artigo será apresentado da seguinte maneira. Na seção 2 são apresentadas características do setor sucroalcooleiro, medidas de risco e estratégias de hedging. Na seção 3 é apresentado o modelo proposto, na seção 4 são apresentados os resultados obtidos com a aplicação do modelo utilizando dados dos mercados brasileiro e americano e finalmente na seção 5 são apresentadas as conclusões do trabalho.

\section{RISCO E HEDGING NO MERCADO SUCROALCOOLEIRO}

Com o crescimento do uso de biocombustíveis em nível mundial, a decisão por parte de produtores tornou-se ainda mais complexa. Além da incerteza inerente à própria atividade de produção agrícola e resultante de características biológicas, variações climáticas e incapacidade de atender a grandes flutuações de demanda, é necessário analisar preços em dois mercados simultaneamente: 0 de alimentos e 0 de energia. Esse é o caso do setor sucroalcooleiro brasileiro. $O$ expressivo aumento da demanda de álcool resultante do crescimento da frota de veículos bicombustível, e que afeta os preços, bem como a dependência dos preços do açúcar de variáveis exógenas, exige que se utilize mecanismos mais sofisticados para gestão de riscos financeiros.

Em linhas gerais a produção açúcar e de álcool apenas difere nos passos após a moagem da cana-de-açúcar (MOREIRA e GOLDEMBERG, 1999). Além do açúcar, do álcool anidro e álcool hidratado, há outros subprodutos que podem ser fabricados a partir da cana-de-açúcar, como a aguardente e o álcool farmacêutico, porém que não representam volumes financeiros expressivos em relação à produção total.

A construção de estratégias de proteção financeira no mercado de derivativos é uma prática comum entre os diversos agentes dos mercados de commodities agrícolas, em particular no mercado de açúcar e álcool. Construir uma estratégia de hedging refere-se à determinação de carteiras de investimento contendo o ativo (no caso de mercados agrícolas, a commodity) e o derivativo de forma a reduzir o risco de flutuação de valor desta carteira. A origem da construção de estratégias de hedging remonta a década de 60, através do uso do modelo clássico de Markowitz (JOHNSON, 1960). Em linhas gerais determina-se um portfólio de mínimo risco 
contendo o ativo e o derivativo, tendo-se como objetivo determinar a razão de hedge, ou seja, o número de contratos futuros (ou de opções) que deve ser adquirido ou vendido de forma a minimizar o risco do investidor. A escolha da medida de risco a ser minimizada é que define a estratégia adotada.

\subsection{Medidas de Risco}

Muitos trabalhos que lidam com seleção de ativos, adotam medidas de risco incapazes de representar a real natureza dos eventos adversos, uma vez que não analisam as caudas das distribuições de perda. Esse é o caso dos modelos que utilizam a variância ou mesmo o valor em risco ( $\mathrm{VaR}$ ) como medida de risco. Exemplos de trabalhos que utilizam esta abordagem são MARTíNEZ-DE-ALBÉNIZ \& SIMCHI-LEVI (2006), KLEINDORFER \& LI (2005) e CHEN et al. (2014).

Os primeiros modelos para composição de portfólios empregavam a variância como medida de risco (MARKOWITZ, 1952; ÇELIKYURT \& ZEKICI, 2007 e ROQUES et al., 2008). Embora tenha seu uso disseminado, a variância apresenta deficiências. Destaca-se o fato de que a variância não analisa as caudas da distribuição de probabilidade dos retornos. OGRYCZAK \& RUSZCZYNSKI (1999) afirmam também que a variância não é capaz de modelar retornos assimétricos.

Como forma de atender a necessidade dos investidores de analisar situações adversas como as que são expressas pelas caudas das distribuições de probabilidade dos retornos das carteiras, o VaR tornou-se com o passar do tempo um instrumento bastante conhecido e utilizado (YOU \& DAIGLER, 2007). O VaR de uma carteira de ativos pode ser definido como a perda máxima do valor de mercado da carteira em um determinado período de tempo com um determinado nível de confiança. Ou seja, por ser uma medida de risco de perda potencial máxima, o VaR nos oferece informação sobre a cauda inferior da distribuição dos retornos do portfólio de ativos, ao contrário da variância. Mas, assim como a variância, o VaR também possui deficiências que podem comprometer a decisão tomada por gestores de ativos, dentre elas o fato de não ser uma medida de risco coerente (ARTZNER et al., 1999). Além disso, o VaR não é uma função convexa em relação à composição da carteira, o que dificulta a otimização na seleção de ativos (RIBEIRO \& FERREIRA, 2005).

Revista Produção Online, Florianópolis, SC, v.14, n. 4, p. 1270-1291, out./dez. 2014. 
Face à necessidade de se criar uma medida de risco que analisasse o risco resultante das caudas de distribuições de probabilidade, ROCKAFELLAR \& URYASEV (2002) criaram uma nova medida denominada Valor em Risco Condicional ou CVaR (Conditional Value-at-Risk). O CVaR mede a perda esperada na cauda da distribuição de perdas um certo portfólio, além do VaR desse portfólio, para um dado nível de confiança. Em outras palavras, o CVaR define o valor médio das perdas da carteira, dado que o pior cenário já ocorreu.

Artigos que buscam selecionar portfólios tendo como medida de risco o CVaR ou M-CVaR (Mean Conditional Value-at-Risk) têm ganho espaço, como pode ser observado em HARRIS e MAZIBAS (2013), HUANG et al. (2010) e WAN et al. (2014). Adicionalmente, KOLM et al. (2014) afirmam que o CVaR é uma das mais populares medidas coerentes de risco utilizada na construção de portfólios atualmente. MANSINI et al. (2014) afirmam modelos de programação linear baseados em CVaR tiveram grande impacto nos novos desenvolvimentos para otimização de portfólio na primeira década do século XXI.

Além de ser uma medida coerente de risco (ABAD et al., 2014) o CVaR, quando utilizado em problemas de seleção de portfólios, possibilita o uso de técnicas de programação linear, o que simplifica expressivamente a resolução do modelo. De acordo com FABOZZI et al. (2007) o CVaR é definido como segue:

$$
\mathrm{CVaR}_{1-\beta}(x)=E\left(f(x, S) \mid f(x, S)>\operatorname{VaR}_{1-\beta}(x)\right)
$$

em que $\mathrm{f}(\mathrm{x}, \mathrm{s})$ representa a função de perda de um portfólio com variável de decisão $\mathrm{x} \in \mathbb{R}^{\mathrm{n}}$ e variável aleatória $\mathrm{S} \in \mathbb{R}^{\mathrm{d}}$. Do mesmo modo que definido no $\mathrm{VaR}$, o nível de confiança é definido por $1-\beta$, comumente sendo $90 \%$, $95 \%$ ou $99 \%$.

Supondo que todas as variáveis aleatórias sejam contínuas, a probabilidade que a função de perda não exceda um certo valor $V a R$, é dada pela equação 2.

$$
\operatorname{CVaR}_{1-\beta}(x)=(1-\beta)^{-1} \int_{f(x, S) \geq \operatorname{VaR}_{1-\beta}(x)} f(x, S) p(S) d S
$$

Contudo, a complexidade deste modelo é grande e dificulta sua aplicação prática. Felizmente, uma abordagem mais simples foi proposta por ROCKAFELLAR Revista Produção Online, Florianópolis, SC, v.14, n. 4, p. 1270-1291, out./dez. 2014. 
\& URYASEV (2002), na qual é possível obter simultaneamente o CVaR e o VaR do portfólio, resolvendo um problema de otimização, dado pela equação 3.

$$
\min _{\left(x_{i} \alpha\right)} F_{\beta}(x, \alpha)=\min _{(x, \alpha)}\left(\alpha+\frac{1}{(1-\beta)} \int_{f(x, S) \geq \operatorname{VaR}_{1-\beta}(x)}(f(x, S)-\alpha) p(S) d S\right)
$$

Na prática a função densidade de probabilidade $\mathrm{p}(\mathrm{S})$ não é conhecida, ou é difícil de estimar. Sendo assim, ROCKAFELLAR \& URYASEV (2002) discretizam o modelo através da definição de $m$ diferentes cenários que são obtidos como uma amostra da função de densidade de probabilidade $\mathrm{S}=\left\{\mathrm{S}_{1}, \ldots, \mathrm{S}_{\mathrm{m}}\right\}$, ou através de simulação computacional. Reescrevendo a equação 3 considerando cenários, temse:

$$
\min _{\left(x_{i} \alpha\right)}\left(\alpha+\frac{1}{m(1-\beta)} \sum_{i=1}^{m} \max \left(f\left(x, S_{i}\right)-\alpha, 0\right)\right)
$$

Substituindo $\max \left(\mathrm{f}\left(\mathrm{x}, \mathrm{S}_{\mathrm{i}}\right)-\alpha, 0\right)$ por variáveis auxiliares $\mathrm{Y}_{\mathrm{i}}$, juntamente com as restrições que possibilitam a linearização do modelo, se obtém o problema de otimização, dado por:

$$
\min _{\left(x_{i} \alpha\right)} \alpha+\frac{1}{m(1-\beta)} \sum_{i=1}^{m} Y_{i}
$$

Com restrições:

(1) $Y_{i} \geq 0, \forall i \in\{1, \ldots, m\}$;

(2) $\quad Y_{i} \geq f\left(x, S_{i}\right)-\alpha, \forall i \in\{1, \ldots, m\}$;

Desse modo, $\mathrm{f}\left(\mathrm{x}, \mathrm{S}_{\mathrm{i}}\right)$ é linear, se chega a um problema linear que pode ser resolvido de modo eficiente com técnicas de programação linear.

\subsection{Estratégias de hedging com futuros e opções}

O hedging permite que se minimize a exposição ao risco de mercado sem que isto conduza à redução do lucro e pode ser realizado com uma grande variedade de instrumentos no mercado de derivativos. Nos casos mais comuns, a proteção é feita com contratos futuros ou de opções.

Revista Produção Online, Florianópolis, SC, v.14, n. 4, p. 1270-1291, out./dez. 2014. 
No caso de commodities agrícolas quando o hedger (quem realiza a operação de hedging) é o produtor, o objetivo é se proteger de uma eventual queda do preço de seu produto no mercado físico. Por isso realiza uma operação no mercado de derivativos que se beneficiará da queda do preço do ativo. Ou seja, quando o preço do ativo cai, o produtor perde na venda do seu produto, mas em compensação ganha no mercado financeiro.

O hedging, quando construído com contratos futuros, limita tanto a perda quanto o ganho de quem o opera. Adotando a notação proposta por CHEN et al. (2003), sejam $S_{t}$ e $F_{t}$ respectivamente, os preços a vista e futuro de um ativo na data $t \in\{0,1\}$ e considere $N_{s}$ o número de unidades disponível da commodity e $N_{f}$ o número de contratos futuros utilizado para realizar o hedging. A variação do valor do portfólio, $\delta V$, composto por ativo e contrato futuro é apresentada na equação 2.

$$
\delta V=N_{S}(\delta S)-N_{f}(\delta F)
$$

em que $\delta S$ representa o retorno do ativo $\boldsymbol{Q}_{1}-S_{0}$ e e $\delta F$ representa o retorno do contrato futuro $F_{1}-F_{0}^{-}$.

O quociente $h=\frac{N_{f}}{N_{S}}$, denominado razão de hedging, é o que se busca encontrar. Uma operação de hedging perfeito consiste em comprar (ou vender) no mercado futuro exatamente o volume disponível no ativo, ou seja, $h=1$.

De maneira mais abrangente ALEXANDER et al. (2006) definem o retorno do portfólio contendo ativos, contratos futuros e opções, como apresentado na equação 3.

$$
\pi(X)=(\delta V)^{T} X
$$

Em que, $\delta V=V_{t}-V_{0}$, é a variação do valor do portfólio, sendo $\delta V=\left[\begin{array}{l}\delta S \\ \delta F \\ \delta O\end{array}\right]$, tal que $\delta S$ é o vetor dos retornos dos ativos negociados no mercado à vista, $\delta F$ o vetor de retornos de contratos futuros e $\delta O$ o vetor dos retornos dos contratos de opções. 
O vetor $\mathrm{X}$ é composto por proporções $x_{i}$ do capital total para cada ativo $i$, mais as razões de hedge associadas a cada derivativo.

Uma opção é um direito, mas não uma obrigação de comprar (ou vender) um ativo em uma data futura, ou de vencimento, por um preço previamente estabelecido, ou preço de exercício K (HULL, 2005). Esse direito é adquirido através do pagamento de um prêmio $p$, que é o preço da opção. No caso de uma opção de compra (call), o detentor da opção tem o direito de comprar o ativo e no caso de uma opção de venda (put), tem o direito de vender o ativo.

A determinação do prêmio da opção de compra $c$ pode ser encontrada através do modelo de Black e Scholes (HULL, 2005).

$c=S_{0} N\left(d_{1}\right)-K e^{-r(T)} N\left(d_{2}\right)$, onde:

$d_{1}=\frac{\ln (S / K)+\left(r+\frac{\sigma^{2}}{2}\right)(T-t)}{\sigma \sqrt{T-t}}, \mathrm{e}$

$d_{2}=d_{1}-\sigma \sqrt{T-t}$

Em que $r$ é a taxa livre de risco, $T$ é a data de vencimento do contrato, $t$ é o tempo atual e $\sigma$ é o desvio padrão dos retornos logarítmicos do ativo, a volatilidade. A função $N(x)$ denota a função de distribuição acumulada normal padrão.

Com o intuito de definir a razão de hedge, para um determinado portfólio, ALEXANDER et al. (2006) apresentam um modelo que minimiza o CVaR (Conditional Value-at-Risk), de um portfólio contendo ativos, futuros e opções, considerando para isso um conjunto de retornos com $m$ amostras ou cenários.

$$
\min _{(x, \alpha)}\left(\alpha+\frac{1}{m(1-\beta)} \sum_{i=1}^{m}\left[-(\partial V)_{i}^{T} x-\alpha\right]^{+}\right)
$$

Sujeito a:

$$
\begin{aligned}
& \left(V^{0}\right)^{T} x=1 ; \\
& (\partial \bar{V})^{T} x=\bar{g} ;
\end{aligned}
$$


$l_{j} \leq x \leq u_{j}, \forall j \in \mathbb{1}_{7} . ., n$

Em que, $\bar{g}$ representa o retorno mínimo exigido para o portfólio, $l_{j}$ e $u_{j}$ representam os limites inferior e superior para a proporção de cada ativo $j$, contrato futuro ou opção, $n$ representa o número total de ativos, e, $\delta \bar{V}$ representa o retorno médio esperado para cada ativo, contrato futuro ou opção.

\section{MODELO PROPOSTO}

O modelo proposto neste artigo constrói estratégias de hedging do produtor de açúcar e álcool, considerando as restrições de produção. O modelo é analisado em duas situações: supondo que se realizam operações de hedging apenas com contratos futuros e também no caso em que se permite o uso de opções como instrumento de hedge.

O produtor de açúcar brasileiro enfrenta alguma dificuldade na realização de hedging utilizando a Bolsa de Mercadorias e Futuros brasileira, uma vez que os derivativos de açúcar possuem pouca liquidez. O produtor pode fazer sua operação de hedging com derivativos de açúcar negociados na Intercontinental Exchange (ICE), contudo o hedging não é perfeito uma vez que os derivativos de açúcar negociados na ICE refletem as variações do preço açúcar de exportação demerara, e não do branco, que é vendido no mercado interno. Os produtores que operam no mercado financeiro, não têm a intenção de realmente fazer a entrega do produto. Dessa maneira, eles costumam liquidar a posição antes do vencimento e nessa operação podem ocorrer perdas e custos de transação. Cabe destacar ainda a quase inexistência de operações de derivativos de álcool, devido à reduzida liquidez desse mercado.

Ao considerar uma carteira contendo açúcar e álcool, bem como contratos futuros e de opção de açúcar, a expressão que descreve do retorno do produtor no final do período será:

$$
\pi\left(x, S_{t}\right)=(\delta V)^{T} X
$$

Em que: 
$X=\left[\begin{array}{c}x_{\text {su }} \\ x_{\text {ani }} \\ x_{\text {hid }} \\ h_{f, s u} \\ h_{o, s u}\end{array}\right]$

$\delta V=\left[\begin{array}{c}\delta S \\ \delta A_{\text {ani }} \\ \delta A_{\text {hid }} \\ \delta F \\ \delta O\end{array}\right]$

$\delta S=\frac{S_{1}-C_{S}}{S_{1}}$

$\delta A_{\text {ani }}=\frac{A_{\text {ani }}-C_{\text {ani }}}{A_{\text {ani }}}$

$\delta A_{\text {hid }}=\frac{A_{\text {hid }}-C_{\text {hid }}}{A_{\text {hid }}}$

$\delta F=\frac{F_{0}-F_{1}}{F_{0}}$

$\delta O=\frac{v-c}{c}$

$\delta S$ é o retorno com a venda de açúcar;

$S_{1}$ é o preço do açúcar no mercado físico previsto para a data $t_{1}$;

$C_{s}$ é o custo de produção do açúcar;

$\delta A_{\text {ani }}$ é o retorno com a venda de etanol anidro;

$A_{a n i}$ é o preço do álcool anidro no mercado físico previsto para a data $t_{1}$;

$C_{a n i}$ é o custo de produção de etanol anidro;

$\delta A_{\text {hid }}$ é o retorno com a venda de etanol hidratado;

$A_{\text {hid }}$ é o preço do álcool hidratado no mercado físico previsto para a data $t_{1}$;

$C_{\text {hid }}$ é o custo de produção de etanol hidratado;

$\delta F$ é o retorno com a utilização de contratos futuros;

$F_{0}$ é o preço do contrato futuro na data inicial $t_{0}$;

$F_{1}$ é o preço do contrato futuro na data final $t_{1}$;

$\delta O$ é o retorno com a utilização de opções de compra (call);

$c$ é o prêmio da opção de venda (call), determinado conforme equação 5;

$v$ é o ganho com a opção na data final $t_{1}$, conforme equação 4;

As variáveis de decisão são:

Revista Produção Online, Florianópolis, SC, v.14, n. 4, p. 1270-1291, out./dez. 2014. 
$x_{s u}:$ a quantidade de açúcar produzido em relação à produção total (\%)

$x_{a n i}:$ a quantidade de etanol anidro produzido em relação à produção total (\%)

$x_{\text {hid }}$ : a quantidade de etanol hidratado produzido (\%)

$h_{f, s u}:$ a razão de hedging com futuros de açúcar

$h_{o, s u}$ : é a proporção do hedging destinada às opções de açúcar;

Observe que não houve a inclusão de derivativos de álcool devido ao risco de liquidez, embora do ponto de vista formal isto pudesse ser considerado. As razões de hedging - os valores $h_{f, s u}$ e $h_{o, s u}$ - são variáveis de decisão, podem assumir qualquer valor entre 0 (zero) e 1 (um), e indicam a porcentagem da produção que está protegida com instrumentos derivativos. LAPAN et al. (1991) mostram, sob hipóteses restritivas a respeito da relação entre preços a vista e futuros, que para uma carteira com um único ativo, o hedging ótimo não inclui opções.

O modelo aqui proposto considera restrições de produção de álcool e açúcar e determina a razão de hedge através da maximização de uma utilidade, levando em conta risco e retorno; ALEXANDER et al. (2006) construíram um modelo similar para a seleção de derivativos para um único ativo e sem considerar o mix de produção.

A razão de hedging será obtida através da maximização da utilidade, considerando-se como medida de risco o CVaR, de tal forma que a função a ser maximizada é $\eta C V a R(X)-E(\pi(X))$ onde o parâmetro de aversão ao risco $\eta$ mede a propensão ao risco do produtor.

O modelo consiste então em minimizar $\eta C V a R(X)-E(\pi(X))$ onde $X$ obedece às seguintes restrições de produção:

(1) $\quad 0 \leq h_{f, s u}+h_{o, s u} \leq x_{s u}$;

(2) $\quad x_{s u}+x_{\text {ani }}+x_{\text {hid }}=1$;

(3) $\quad l i_{s u} \leq x_{s u} \leq l s_{s u}$;

(4) $\quad l i_{a n i} \leq x_{a n i} \leq l s_{a n i}$;

(5) $\quad l i_{\text {hid }} \leq x_{\text {hid }} \leq l s_{\text {hid }}$;

(6) $\quad l i_{\text {eta }} \leq x_{\text {ani }}+x_{\text {hid }} \leq l s_{\text {eta }}$.

Revista Produção Online, Florianópolis, SC, v.14, n. 4, p. 1270-1291, out./dez. 2014. 
Em que $l i_{s u}, l i_{a n i}, l i_{\text {hid }}$ e $l i_{\text {eta }}$ são os limites inferiores para a produção de açúcar, álcool anidro, álcool hidratado, e etanol como um todo. Assim como $l s_{s u}, l s_{a n i}, l s_{\text {hid }}$ e $l s_{\text {eta }}$ são os limites superiores para a produção dos mesmos produtos.

A restrição 1 restringe a razão de hedge ao volume total a ser dividido entre diversos produtos, eliminando a possibilidade de o produtor atuar no mercado de derivativos como especulador. A restrição 2 garante que o modelo distribua toda a produção entre açúcar, etanol anidro e etanol hidratado. As restrições 3-6 garantem que a capacidade de produção das usinas não seja excedida tanto para o açúcar como para o etanol.

O modelo para determinação do portfólio considerando açúcar, álcool anidro, álcool hidratado, contratos futuros de açúcar e opções de venda de açúcar, é dado pela equação 13.

$\min _{\left(\beta_{i} \alpha\right)} \alpha+\frac{1}{m(1-\beta)} \sum_{i=1}^{m} Y_{i}$

Com restrições:
(1) $\pi\left(x, S_{t}\right) \geq R$
(2) $\quad Y_{i} \geq 0, \forall i \in\{1, \ldots, m\}$;
(2) $Y_{i} \geq \pi_{i}(X)-\alpha, \forall i \in\{1, \ldots, m\}$;
(3) 6 restrições de produção, conforme equações 18

Em que os parâmetros de entrada são:

$\pi(X)$ representa o retorno do portfólio no cenário $i$, conforme equação 10 ;

$R$ é o retorno mínimo desejado;

$m$ é o número de cenários de preços passados;

$\beta$ é o nível de confiança

$\alpha$ representa o VaR (Value-at-Risk), definido pelo próprio modelo;

$Y_{i}$ são variáveis auxiliares utilizadas para linearizar o modelo. 


\section{RESULTADOS}

Para analisar o modelo, foram empregados dados a respeito do mercado sucroalcooleiro nacional. Os custos de produção do açúcar, do etanol anidro e do etanol hidratado são considerados na função objetivo. Os dados de custo de produção são levantados pela ESALQ (Escola Superior de Agricultura Luiz de Queiroz). O modelo utiliza preços históricos de açúcar, álcool e derivativos de açúcar (futuros e opções) como parâmetros de entrada do modelo. É assumido neste trabalho que preços futuros tendem a se comportar de maneira semelhante aos preços observados no passado.

Os dados utilizados vão de Setembro de 2000 a Junho de 2010, num total de 40 instantes de tempo que coincidem com os vencimentos dos contratos futuros de açúcar. Os preços físicos do açúcar, do etanol anidro e do etanol hidratado são disponibilizados pela ESALQ. Os preços dos contratos futuros de açúcar foram obtidos da bolsa ICE (Intercontinental Exchange).

Os preços do açúcar e do etanol foram convertidos para dólar por ATR (Açúcar Total Recuperável), que é a quantidade correspondente de matéria-prima necessária para gerar o açúcar ou etanol. Os fatores de conversão para ATR são apresentados na Tabela 1.

Tabela 1 - Fatores de conversão dos para preços em US\$/ATR, Fonte: UNICA

\begin{tabular}{ccc}
\hline Açúcar & Etanol Anidro & Etanol Hidratado \\
\hline 1.0495 & 1.7651 & 1.6913 \\
\hline
\end{tabular}

A decisão sobre como dividir a produção entre açúcar e álcool depende, entre outros fatores, de restrições de produção. Nas simulações realizadas foi considerado que é possível dividir a produção em qualquer combinação entre os três produtos (etanol hidratado, etanol anidro e açúcar). Contudo não é incomum encontrar restrições em usinas que produzem apenas etanol ou precisam produzir pelo menos $40 \%$ de açúcar em função de restrições operacionais.

Quatro cenários reais e distintos foram selecionados. Em cada cenário os retornos esperados com a venda de cada subproduto, com as operações no mercado futuro e de opções foram levantados. Foram selecionados cenários em

Revista Produção Online, Florianópolis, SC, v.14, n. 4, p. 1270-1291, out./dez. 2014. 
diferentes datas que alternassem a preferência entre cada subproduto, de forma a evidenciar o processo de decisão do modelo. A Tabela 2 apresenta os cenários escolhidos e os retornos ocorridos para cada ativo considerado.

Tabela 2 - Cenários escolhidos e retornos ocorridos

\begin{tabular}{ccccccc}
\hline Cenário & Data & Açúcar & $\begin{array}{c}\text { Etanol } \\
\text { Anidro }\end{array}$ & $\begin{array}{c}\text { Etanol } \\
\text { Hidratado }\end{array}$ & $\begin{array}{c}\text { Futuro } \\
\text { de } \\
\text { Açúcar }\end{array}$ & $\begin{array}{c}\text { Opções } \\
\text { de } \\
\text { Açúcar }\end{array}$ \\
\hline 01 & $28 / 09 / 2001$ & $17.8 \%$ & $23.5 \%$ & $26.5 \%$ & $6.5 \%$ & $4.4 \%$ \\
02 & $30 / 06 / 2004$ & $17.4 \%$ & $14.4 \%$ & $12.6 \%$ & $-2.2 \%$ & $0.6 \%$ \\
03 & $30 / 09 / 2004$ & $19.1 \%$ & $28.0 \%$ & $23.8 \%$ & $-4.5 \%$ & $-1.6 \%$ \\
04 & $29 / 09 / 2006$ & $34.9 \%$ & $39.9 \%$ & $34.7 \%$ & $1.5 \%$ & $5.3 \%$ \\
\hline
\end{tabular}

No primeiro cenário ocorrido em 28/09/2001, o retorno do etanol hidratado é maior que o retorno do etanol anidro que por sua vez supera o retorno do açúcar. Já os retornos dos contratos futuros e também das opções são positivos. Duas simulações foram feitas, a primeira considerando a utilização de opções e de contratos futuros e a segunda considerando apenas a utilização de contratos futuros como instrumento de hedge.

A Figura 1 apresenta os gráficos obtidos da simulação do primeiro cenário. No gráfico da fronteira eficiente o termo "FE - Opt" representa a fronteira eficiente para a solução com opções, enquanto o termo "FE - No opt" representa a fronteira eficiente para a solução sem opções. No gráfico razão de hedge o termo "ho" representa a proporção de opções na constituição da carteira com opções e futuros, "hf - opt" representa a proporção de contratos futuros na constituição da carteira com opções e futuros, e "hf - NO opt" representa a proporção de contratos futuros na constituição da carteira que utiliza apenas contratos futuros para fins de hedge. Os termos "ws", "wea" e "weh", nos gráficos de mix de produção, com e sem opções, representam a proporção de açúcar, etanol anidro e etanol hidratado respectivamente.

Os gráficos de mix de produção são iguais nas situações com ou sem opções, sendo o açúcar a alternativa escolhida para a situação de menor risco, enquanto para o maior risco possível se produziria apenas etanol hidratado.

O gráfico da fronteira eficiente mostra que as curvas de risco/retorno do modelo com e sem opções são coincidentes. Isso ocorre, pois as opções não são utilizadas para redução do risco, como evidenciado pelo gráfico razão de hedge, 
onde $h_{0}$ que representa o uso de opções se mantém em zero durante todo o intervalo de CVaR.

A solução dada pelo modelo com opções e pelo modelo sem opções coincidem, pois os contratos futuros de açúcar conseguem reduzir o risco e ainda entregar um retorno positivo maior que as opções. Desse modo as opções não trariam benefício ao produtor.

No cenário 4, ocorrido em 29/09/2006, o etanol anidro apresenta o maior retorno, seguido do açúcar e do etanol hidratado. Ao contrário do cenário 1, o retorno com opções de açúcar supera o retorno com futuros de açúcar. Essas características fazem com que as decisões do modelo com opções provoquem resultados diferentes das decisões do modelo sem opções. Uma das diferenças é apresentada pelo gráfico de fronteira eficiente da Figura 2, onde a curva gerada pelo modelo sem opções mostra que não é possível apresentar retornos eficientes para um CVaR maior que cerca de $34 \%$.

As decisões de mix de produção apresentam similaridades entre o modelo com opções e o modelo sem opções. Em ambos os casos se produz $100 \%$ de açúcar na situação de menor risco e se produz $100 \%$ de etanol anidro na situação de maior risco. A diferença entre as curvas está na amplitude do risco, sendo que o modelo sem opções apresenta amplitude de risco menor.

Figura 1 - Gráficos para o Cenário 1

Fronteira Eficiente

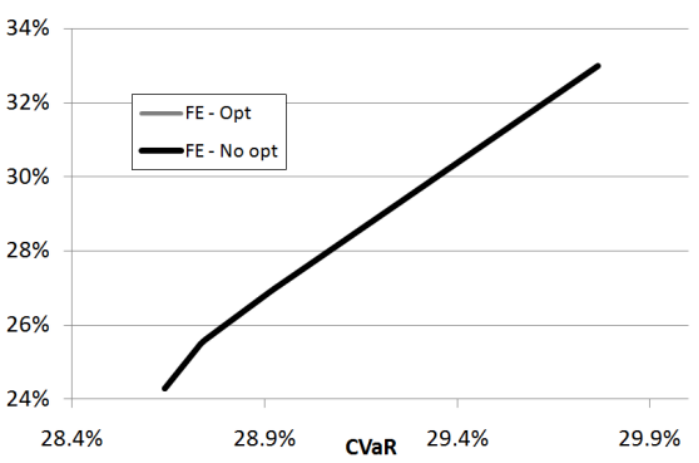

Mix de Produção - Com Opções

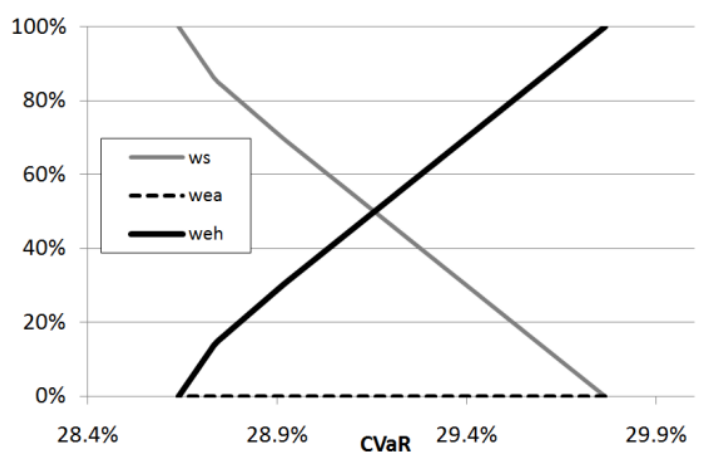



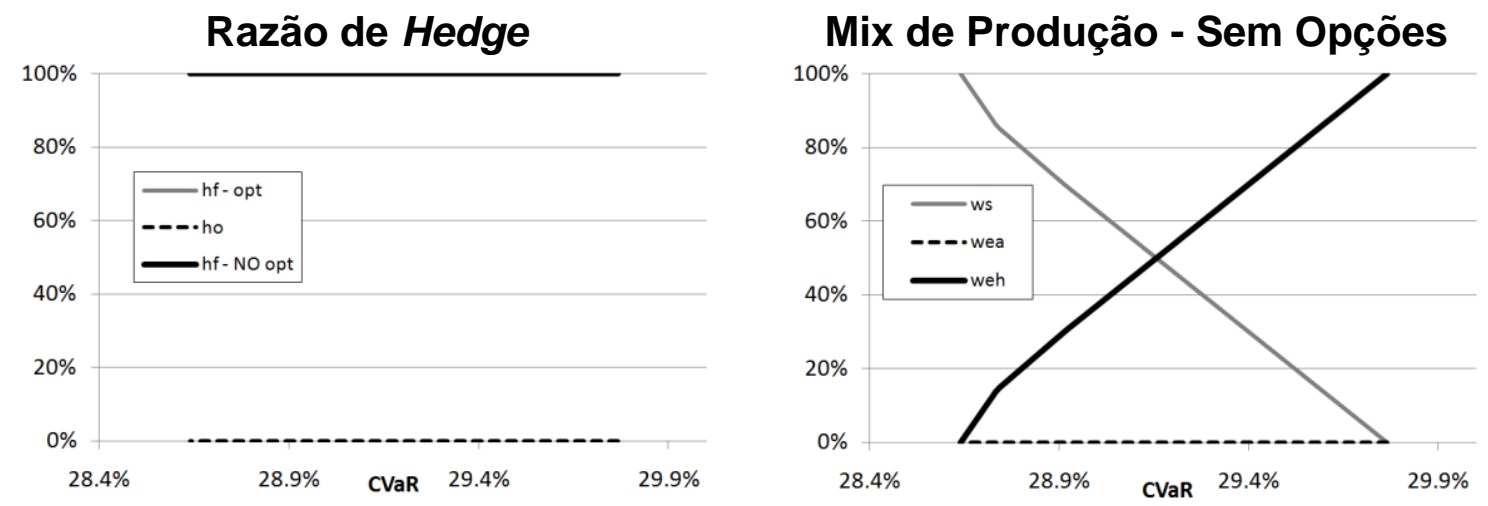

A razão de hedge no modelo com opções é feita totalmente com contratos futuros para a situação de menor risco, e na situação com maior risco é feita totalmente com opções. Já o modelo sem opções, utiliza a razão de hedge de 100\% de contratos futuros, o que significa que toda a produção é protegida através de contratos futuros.

Com isso é possível notar que a utilização de opções para fins de hedge só se justifica quando as opções conseguem reduzir mais eficazmente o risco e ainda apresentam impacto mais positivo no retorno em comparação com os contratos futuros. No cenário 3 onde, similarmente ao cenário 1 , as decisões foram as mesmas em ambos os modelos, com e sem opções, apesar de as opções apresentarem retorno mais favorável, não foram tão eficazes na redução do risco quanto os contratos futuros. Já o cenário 2, apresentou resultado similar ao cenário 4 , pois a relação risco / retorno das opções foi mais favorável que a apresentada pelos contratos futuros.

Figura 2 - Gráficos para o Cenário 4

\section{Fronteira Eficiente}

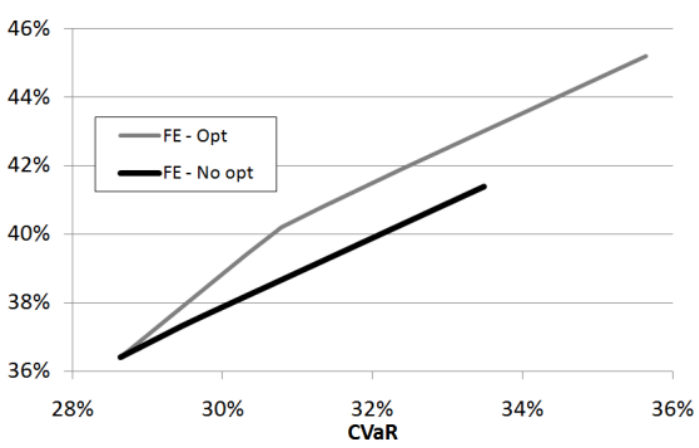

\section{Mix de Produção - Com Opções}

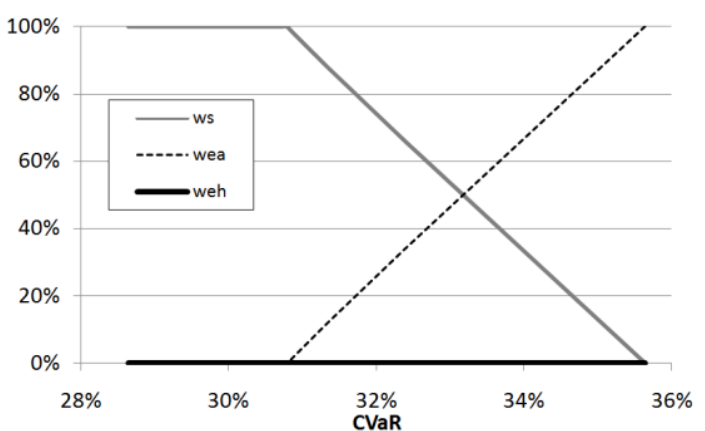



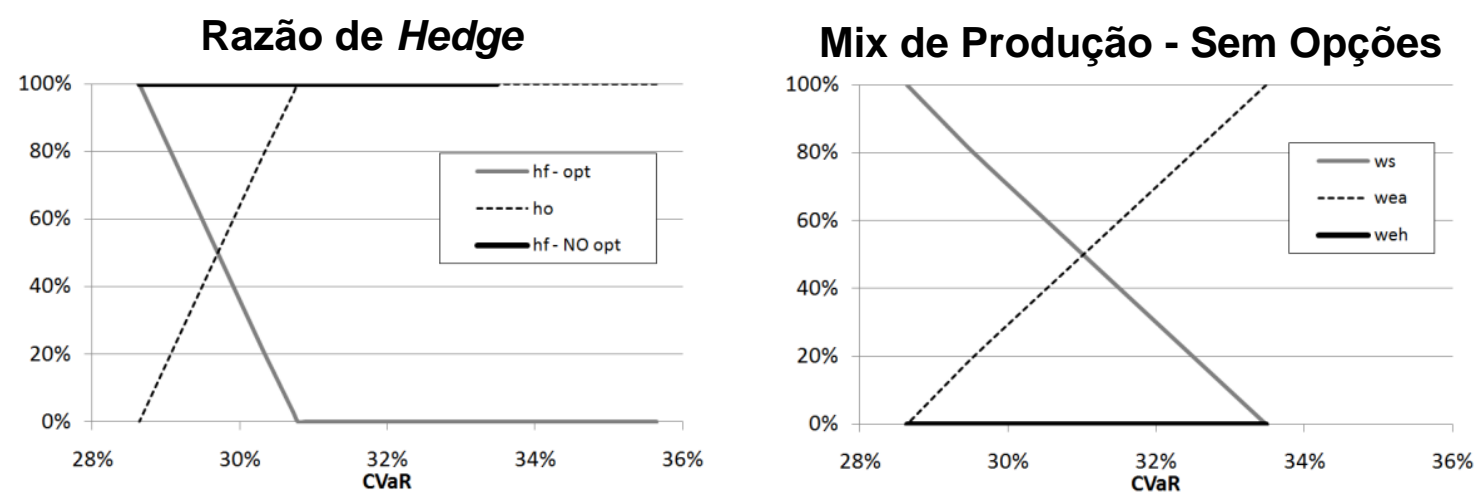

LAPAN et al. (1991) concluíram que quando preços futuros e prêmios de opções não são viesados as opções geram resultados redundantes aos resultados obtidos com futuros. Contudo os autores atentam para a presença de risco de base, ou seja, risco associado às imperfeições no comportamento dos preços futuros e dos prêmios de opções, seja por descasamento de prazos, pela utilização de contratos de commodities não idênticas as que se deseja proteger, ou ainda diversos outros fatores ligados às incertezas de mercado. Nos casos em que o risco de base é suficientemente grande a diferença entre os preços futuros e à vista, podem ser grandes o suficiente para que opções e contratos futuros gerem resultados diferentes. Essas diferenças obviamente levam a resultados diferentes nos modelos que consideram opções e futuros em relação aos modelos que consideram apenas futuros, como demonstrado pelo presente artigo.

\section{CONCLUSÕES}

Uma das contribuições desse trabalho foi a criação de um modelo capaz de construir a fronteira eficiente para um produtor de açúcar e álcool que busca derivativos como instrumentos de hedge. O modelo proposto é capaz de definir o mix de produção e a razão de hedge ideais para um produtor, de acordo com sua tolerância ao risco.

Uma segunda contribuição foi a constatação de que a utilização de opções como instrumentos de hedge pode alterar de maneira significativa a fronteira eficiente para um produtor que utiliza apenas futuros. Esse resultado reforça a 
constatação de LAPAN et al. (1991), de que opções podem afetar os resultados de decisões de portfólio desde que o risco de base seja suficientemente grande. Desse modo um produtor pode se beneficiar do uso de opções, principalmente se conseguir prever o comportamento do risco de base.

Ainda existe um grande espaço para pesquisas futuras, principalmente pelo fato de as decisões serem altamente dependentes da capacidade de prever a evolução dos preços, portanto modelos mais sofisticados de previsão podem ser associados ao modelo de decisão de mix. Outro fator importante é que o modelo proposto não é capaz de decidir sobre estocagem, uma decisão comum aos produtores que podem se beneficiar da sazonalidade. E ainda a possibilidade de diversificação não é exclusividade do mercado sucroalcooleiro, portanto seria relevante para a validade do modelo testá-lo para outros mercados.

\section{REFERÊNCIAS}

ABAD, P.; BENITO, S. e LÓPEZ, C. A comprehensive review of Value at Risk methodologies. The Spanish Review of Financial Economics, v.12, p.15-32, 2014. http://dx.doi.org/10.1016/j.srfe.2013.06.001

AITSAHLIA, F.; WANG, C.; CABRERA V. E.; URYASEV, S. e FRAISSE, C. W. Optimal crop planting schedules and financial hedging strategies under ENSO-based climate forecasts. Annals of Operations Research, 2009.

ALEXANDER, S.; COLEMAN, T.F.; LI, Y. Minimizing CVaR and VaR for a portfolio of derivatives. Journal of Banking and Finance, v.30, p.583-605, 2006.

http://dx.doi.org/10.1016/i.jbankfin.2005.04.012

ARTZNER, P.; DELBAEN, F.; EBER, J. M.; HEATH, D. Coherent measures of risk. Mathematical Finance, v.9, n.3, p.203-228, 1999. http://dx.doi.org/10.1111/1467$\underline{9965.00068}$

BASTIAN-PINTO. C.; BRANDÃO, L. HAHN, W. J. Flexibility as a source of value in the production of alternative fuels: the ethanol case. Energy Economics, v.31, p.411-422, 2009. http://dx.doi.org/10.1016/j.eneco.2009.02.004

CHEN, R.; LI, C.; WANG, W. e WANG, Z. Empirical analysis on future-cash arbitrage risk with portfolio VaR. Physica A, v.398, p.210-216, 2014.

http://dx.doi.org/10.1016/i.physa.2013.12.017

CHEN, S.S.; LEE, C.F.; SHRESTHA, K. Futures hedge ratios: a review. The

Quarterly Review of Economics and Finance, v.43, p.433-465, 2003.

http://dx.doi.org/10.1016/S1062-9769(02)00191-6

Revista Produção Online, Florianópolis, SC, v.14, n. 4, p. 1270-1291, out./dez. 2014. 
CHEN, S.S.; LEE, C.F.; SHRESTHA, K. Do the pure martingale and joint normality hypotheses hold for futures contracts? Implications for the optimal hedge ratiosging with forwards and puts in complete and incomplete markets. The Quarterly Review of Economics and Finance, v.48, p.153-174, 2008.

http://dx.doi.org/10.1016/i.gref.2005.10.002

ÇELIKYURT, U. \& SÜLEYMAN ÖZEKICI, S. Multiperiod portfolio optimization models in stochastic markets using the mean-variance approach. European Journal of Operational Research, v. 179, n. 1, p. 186-202, 2007. http://dx.doi.org/10.1016/i.ejor.2005.02.079

EBADIAN, M.; SOWLATI, T.; SOKHANSANJ, S. e TOWNLEY-SMITH, L. Modeling and analyzing storage systems in agricultural biomass supply chain for cellulosic ethanol production. Applied Energy, v.102, p.840-849, 2013.

http://dx.doi.org/10.1016/..apenergy.2012.08.049

FABOZZI, F. J.; KOLM, P. N.; PACHAMANOVA, D. A. e FOCARDI, S. M. Robust portfolio optimization and management. 1st ed., Editora: John Wiley \& Sons. Ltd, 2007.

GEMAN, H. \& OHANA, S. Time-consistency in managing a commodity portfolio: A dynamic risk measure approach. Journal of Banking Finance, v. 32, n.10, p.19912005, 2008. http://dx.doi.org/10.1016/j.jbankfin.2007.05.020

HARRIS, R. D. F. \& MAZIBAS, M. Dynamic hedge fund portfolio construction: A semi-parametric approach. Journal of Banking Finance, v. 37, p.139-149, 2013. http://dx.doi.org/10.1016/i.jbankfin.2012.08.017

HUANG, D.; ZHU, S.; FABOZZI, F. J. e FUKUSHIMA, M. Portfolio selection under distributional uncertainty: A relative robust $\mathrm{CVaR}$ approach. European Journal of Operational Research, v. 203, p.185-194, 2010.

http://dx.doi.org/10.1016/i.ejor.2009.07.010

HULL, J. C. Fundamentos dos mercados futuros e de opções. São Paulo: Bolsa de Mercadorias e Futuros, 2005.

JOHNSON, L. L. The theory of hedging and speculation in commodity futures. The Review of Economic Studies, v. 27, p.139-151, 1960.

http://dx.doi.org/10.2307/2296076

KLEINDORFER, P. \& LI, L. Multi-period VaR-constrained portfolio optimization in electric power. The Energy Journal, p. 1-26, 2005.

KOLM, P. N.; TÜTÜNCÜ, R. e FAVOZZI, F. J. 60 Years of portfolio optimization: Practical challenges and current trends. European Journal of Operational

Research, v.234, p. 356-371, 2014. http://dx.doi.org/10.1016/j.ejor.2013.10.060 
LAPAN, H.; MOSCHINI, G.; HANSON, S. Production, hedging and speculative decisions with options and futures markets. American Journal of Agricultural Economics, 1991, v.72, p.66-74, 1991.

MANSINI, R.; OGRYCZAK, W. e SPERANZA, M. G. Twenty years of linear programming based portfolio optimization. European Journal of Operational Research, v.234, p.518-535, 2014. http://dx.doi.org/10.1016/j.ejor.2013.08.035

MARTÍNEZ-DE-ALBÉNIZ, V. \& SIMCHI-LEVI, D. Mean-Variance Trade-offs in Supply Contracts. Naval Research Logistics, n.53, p.603-616, 2006. http://dx.doi.org/10.1002/nav.20186

MARKOWITZ, H. M. Portfolio Selection. Journal of Finance, v.7, p.77-91, 1952. http://dx.doi.org/10.2307/2975974

MOREIRA, J.R.; GOLDEMBERG, J. The alcohol program. Energy Policy, v.27, p.229-245, 1999. http://dx.doi.org/10.1016/S0301-4215(99)00005-1

OGRYCZAK, W., RUSZCZYNSKI, A. From stochastic dominance to mean risk models: Semideviations and risk measures. European Journal of Operational Research, v.116, p.33-50, 1999. http://dx.doi.org/10.1016/S0377-2217(98)00167-2

RIBEIRO, C. O. \& FERREIRA, L. A. Uma contribuição ao problema de composição de carteiras de mínimo valor em risco. Gestão \& Produção, v.12, n.2, p.295-304, 2005. http://dx.doi.org/10.1590/S0104-530X2005000200012

ROCKAFELLAR, R.T.; URYASEV, S. Conditional value at risk for general loss distributions. Journal of Banking and Finance,v.26, p.1443-1447, 2002. http://dx.doi.org/10.1016/S0378-4266(02)00271-6

ROQUES, F.A.; NEWBERY, D.M.; NUTTALL, W.J. Fuel mix diversification incentives in liberalized electricity markets: A Mean-Variance Portfolio theory approach. Energy Economics, V.30, p. 1831-1849, 2008. http://dx.doi.org/10.1016/i.eneco.2007.11.008

VAN DYKEN, S.; BAKKEN, B. H. e SKJELBRED, H. I. Linear mixed-integer models for biomass supply chains with transport, storage and processing. Energy, v.35, p. 1338-1350, 2010. http://dx.doi.org/10.1016/j.energy.2009.11.017

WAN, Y.; CLUTTER, M. L.; MEI, B. e SIRY, J. P. Assessing the role of U.S. timberland assets in a mixed portfolio under the mean-conditional value at risk framework. Forest Policy and Economics, ARTICLE IN PRESS, 2014.

http://dx.doi.org/10.1016/j.forpol.2014.06.002

YOU, L.; DAIGLER, R. Downside Four-Moment Tail Risk and its Effect on Financial and Commodity Diversification. Probabilistic Constrained Optimization, S.P Uryasev, p.272-281, 2000. 
ZHANG, J.; OSMANI, A.; AWADU, I. e GONELA, V. An integrated optimization model for switchgrass-based bioethanol supply chain. Applied Energy, v.102, p.1205-1217, 2013. http://dx.doi.org/10.1016/j.apenergy.2012.06.054

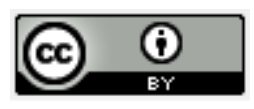

Artigo recebido em 24/03/2013 e aceito para publicação em 26/09/2014 DOI: http://dx.doi.org/ 10.14488/1676-1901.v14i4.1559 